\title{
Über Galoisgruppen lokaler Körper
}

\section{Uwe Jannsen}

Fakultät für Mathematik, Universität Regensburg, Universitätsstr. 31, 8400 Regensburg, Bundesrepublik Deutschland

Der erste Schritt bei der Untersuchung der absoluten Galoisgruppe $G_{k}=G(\vec{k} / k)$ eines lokalen Körpers $k$ besteht nach einer Idee von Iwasawa [7] darin, die Operation der Galoisgruppe $\mathscr{G}=G(T / k)$ der maximalen zahm-verzweigten Erweiterung $T$ von $k$ auf der Faktorkommutatorgruppe $V_{k}^{\text {ab }}$ der Verzweigungsgruppe $V_{k}=G(\bar{k} / T)$ zu betrachten. Nach der Klassenkörpertheorie ist $V_{k}^{\text {ab }} \mathscr{G}_{-}$ isomorph zum projektiven Limes der Einseinheitengruppen $U_{K}^{1}$ der endlichen zahm-verzweigten galoisschen Erweiterungen $K / k$, und Iwasawa beschrieb diese als Moduln über dem Gruppenring $\mathbb{Z}_{p}[G(K / k)]$.

In der vorliegenden Arbeit soll gezeigt werden, daß sich hier durch das Studium kohomologisch trivialer $\mathbb{Z}_{p}[G]$-Moduln einige neue Resultate erhalten lassen. Grundlegend ist dafür der Satz, da $B$ ein derartiger Modul $M$ bereits durch seinen Torsionsmodul Tor $(M)$ und den Modul $\mathbb{Q}_{p} \otimes M$ vollständig bestimmt wird. Für den Fall der Isomorphie $\mathbb{Q}_{p} \otimes M \cong \mathbb{Q}_{p}[G]^{m}$ wird weiter ein konstruktives Verfahren entwickelt, um die Struktur von $M$ aus der von Tor $(M)$ zu gewinnen.

Für eine zahm-verzweigte Erweiterung $K / k$ p-adischer Zahlkörper liefert dies Verfahren aufgrund der wohlbekannten Isomorphie $\mathbb{Q}_{p} \otimes U_{K}^{1} \cong \mathbb{Q}_{p}[G]^{n}$ mit $n=\left[k: \mathbb{Q}_{p}\right]$ und $G=G(K / k)$ eine exakte Sequenz

$$
0 \rightarrow \mathbb{Z}_{p}[G] \rightarrow \mathbb{Z}_{p}[G]^{n+1} \rightarrow U_{K}^{1} \rightarrow 1,
$$

insbesondere erhält man eine Beschreibung von $U_{K}^{1}$ durch eine einzige Relation statt durch zwei wie bei Iwasawa.

Dies hat eine wichtige Konsequenz für die Darstellung der Galoisgruppe $G_{k}$ durch Erzeugende und Relationen. Aus der exakten Sequenz

$$
0 \rightarrow \mathbb{Z}_{p} \llbracket \mathscr{G} \rrbracket \rightarrow \mathbb{Z}_{p} \llbracket \mathscr{G} \rrbracket^{n+1} \rightarrow V_{k}^{\mathrm{ab}} \rightarrow 1
$$

folgt, daß zur Beschreibung von $G_{k}$ neben der Hasse-Iwasawa-Relation, die von $\mathscr{G}$ stammt, nur noch eine weitere Relation nötig ist. (Die bisherigen Untersuchungen von Koch [10] und Jakovlev [8] gehen aufgrund der Iwasawaschen Ergebnisse immer von zwei weiteren Relationen aus.) Genauer wird 
folgendes gezeigt: Bildet man die Gruppe $F(n+1, \mathscr{G})$, indem man im freien proendlichen Produkt von $\mathscr{G}$ mit einer freien pro-endlichen Gruppe $F_{n+1}$ mit $n+1$ Erzeugenden $z_{0}, \ldots, z_{n}$ den von den $z_{i}$ erzeugten Normalteiler zur pro-p-Gruppe macht, so gibt es eine Surjektion

$$
F(n+1, \mathscr{G}) \rightarrow G_{k},
$$

deren Kern als Normalteiler von einem Element $r$ erzeugt wird. In [9] wird dieses $r$ für $p \neq 2$ angegeben, das obige Resultat gilt jedoch auch für $p=2$.

Die entsprechenden Ergebnisse für einen Potenzreihenkörper $k$, die auf Koch [11] zurückgehen, lassen sich zitieren in der Form

$$
V_{k}^{\mathrm{ab}} \cong \mathbb{Z}_{p} \llbracket \mathscr{G} \rrbracket^{\mathbb{N}} \quad G_{k} \cong F(\mathbb{N}, \mathscr{G}) .
$$

Die Verwendung der Gruppen $F(J, \mathscr{G})$ erspart dabei die etwas komplizierte Konstruktion einer freien Operatoren-pro- $p$-Gruppe.

$\mathrm{Da}$ es keine zusätzlichen Schwierigkeiten bereitet, werden allgemeiner die Galoisgruppen $p$-abgeschlossener Erweiterungen betrachtet.

\section{§1. Kohomologisch triviale $\mathbb{Z}_{p}[G]-$ Moduln}

Sei $G$ in diesem Paragraphen eine beliebige endliche Gruppe. Sei $p$ eine Primzahl, $\mathbb{Q}_{p}$ der Körper der $p$-adischen Zahlen und $\mathbb{Z}_{p}$ der Ring der ganzen $p$-adischen Zahlen. Die Moduln $\mathbb{Q}_{p}, \mathbb{Z}_{p}$ und $\mathbb{Q}_{p} / \mathbb{Z}_{p}$ werden immer mit der trivialen $G$-Operation versehen. Für einen kommutativen Ring $R$ bezeichne $R[G]$ den Gruppenring mit Koeffizienten in $R$. Für $\mathbb{Z}_{p}[G]$-Moduln $A$ und $B$ werden $\operatorname{Hom}(A, B):=\operatorname{Hom}_{\mathbb{Z}_{p}}(A, B)$ und $A \otimes B:=A \otimes_{\mathbb{Z}_{p}} B$ zu $\quad \mathbb{Z}_{p}[G]$-Moduln durch die Definitionen $(s f)(a):=s f\left(s^{-1} a\right)$ und $s(a \otimes b):=s a \otimes s b$ für $s \in G$. Insbesondere sei $A^{+}=\operatorname{Hom}\left(A, \mathbb{Z}_{p}\right)$ das $\mathbb{Z}_{p}$-Dual und $A^{*}=\operatorname{Hom}\left(A, \mathbb{Q}_{p} / \mathbb{Z}_{p}\right)$ das Pontrjagindual eines $\mathbb{Z}_{p}[G]$-Moduls $A$. (Bei der Bildung von $A^{*}$ wird $A$ immer endlich erzeugt oder diskret, also auch lokalkompakt sein.) Schließlich sei Tor $(A)$ der Torsionsmodul von $A$.

Die folgenden Eigenschaften projektiver $\mathbb{Z}_{p}[G]$-Moduln sind wohlbekannt.

Lemma 1.1. Seien $P$ und $P^{\prime}$ projektive, endlich erzeugte $\mathbb{Z}_{p}[G]$-Moduln, dann gilt a) $P \cong P^{\prime}$ genau dann, wenn $P / p P^{\prime}$ (s. etwa [5] 3.17).

b) $P \cong P^{\prime}$ genau dann, wenn $\mathbb{Q}_{p} \otimes P \cong \mathbb{Q}_{p} \otimes P^{\prime}$. (Dies ist ein Ergebnis von Swan [16], Cor. 6.4, und wird im folgenden als Lemma von Swan zitiert.)

Dies soll nun auf kohomologisch triviale Moduln verallgemeinert werden. Man beachte hierzu, daß ein endlich erzeugter $\mathbb{Z}_{p}[G]$-Modul genau dann projektiv ist, wenn er kohomologisch trivial und torsionsfrei ist ([14], IX, $\S 5$, Th. 7 mit $\mathbb{Z}_{p}$ statt $\mathbb{Z}$ ).

Satz 1.2. Seien $M$ und $M^{\prime}$ endlich erzeugte, kohomologisch triviale $\mathbb{Z}_{p}[G]$ Moduln, dann sind die folgenden Aussagen äquivalent:

a) $M \cong M^{\prime}$.

b) $\operatorname{Tor}(M) \cong \operatorname{Tor}\left(M^{\prime}\right)$ und $\mathbb{Q}_{p} \otimes M \cong \mathbb{Q}_{p} \otimes M^{\prime}$. 
c) $\operatorname{Tor}(M) \cong \operatorname{Tor}\left(M^{\prime}\right)$ und $M / p M \cong M^{\prime} / p M^{\prime}$.

d) Für ein s mit $p^{s} \operatorname{Tor}(M)=p^{s}$ Tor $\left(M^{\prime}\right)=0$ gilt $M / p^{s+1} M \cong M^{\prime} / p^{s+1} M^{\prime}$.

Beweis. Wegen der kohomologischen Trivialität von $M$ und $M^{\prime}$ gibt es exakte Sequenzen

$$
\begin{aligned}
& 0 \rightarrow P \rightarrow Q \rightarrow M \rightarrow 0 \\
& 0 \rightarrow P^{\prime} \rightarrow Q^{\prime} \rightarrow M^{\prime} \rightarrow 0
\end{aligned}
$$

mit endlich erzeugten, projektiven $\mathbb{Z}_{p}[G]$-Moduln $P, Q, P^{\prime}$ und $Q^{\prime}([14]$, IX, $\S 5$, Th. 8 für $\mathbb{Z}_{p}[G]$-Moduln).

Tensorieren wir diese Sequenzen mit $\mathbb{Q}_{p}$, so zerfallen sie nach dem Satz von Maschke. Dies ergibt die Isomorphien $\mathbb{Q}_{p} \otimes Q \cong \mathbb{Q}_{p} \otimes P \oplus \mathbb{Q}_{p} \otimes M$ und $\mathbb{Q}_{p} \otimes Q^{\prime} \cong \mathbb{Q}_{p} \otimes P^{\prime} \oplus \mathbb{Q}_{p} \otimes M^{\prime}$, insbesondere also

$$
\mathbb{Q}_{p} \otimes\left(P \oplus Q^{\prime}\right) \oplus \mathbb{Q}_{p} \otimes M \cong \mathbb{Q}_{p} \otimes\left(P^{\prime} \oplus Q\right) \oplus \mathbb{Q}_{p} \otimes M^{\prime}
$$

Setzen wir weiter für einen $\mathbb{Z}_{p}[G]$-Modul $A$ und $n \in \mathbb{N}$

$$
\begin{aligned}
& A_{n}=A / n A, \\
& { }_{n} A=\{a \in A ; n a=0\},
\end{aligned}
$$

so erhalten wir mit dem Schlangenlemma aus $(+)$ die exakten Sequenzen

$$
\begin{aligned}
& 0 \rightarrow{ }_{n} \operatorname{Tor}(M) \rightarrow P_{n} \rightarrow Q_{n} \rightarrow M_{n} \rightarrow 0 \\
& 0 \rightarrow{ }_{n} \operatorname{Tor}\left(M^{\prime}\right) \rightarrow P_{n}^{\prime} \rightarrow Q_{n}^{\prime} \rightarrow M_{n}^{\prime} \rightarrow 0 .
\end{aligned}
$$

Wir kommen nun zum eigentlichen Beweis des Satzes.

a) $\Rightarrow$ d) ist trivial.

d) $\Rightarrow$ c). Offenbar gilt $\quad{ }_{p}\left(M / p^{s+1} M\right)=\left({ }_{p} \operatorname{Tor}(M)+p^{s} M\right) / p^{s+1} M \quad$ und also ${ }_{p}\left(M / p^{s+1} M\right) / p^{s}\left(M / p^{s+1} M\right) \cong{ }_{p} \operatorname{Tor}(M)$ wegen $\operatorname{Tor}(M) \cap p^{s} M=0$. Aus d) folgt daher ${ }_{p} \operatorname{Tor}(M) \cong{ }_{p} \operatorname{Tor}\left(M^{\prime}\right)$. Andererseits folgt aus d) auch $M_{p} \cong M_{p}^{\prime}$, mit $(+++)$ und dem Lemma von Schanuel (s. [16]; zweimalige Anwendung, vg1. [4], S. 163) also

$$
{ }_{p} \operatorname{Tor}(M) \oplus P_{p}^{\prime} \oplus Q_{p} \cong{ }_{p} \operatorname{Tor}\left(M^{\prime}\right) \oplus P_{p} \oplus Q_{p}^{\prime} .
$$

Zusammen ergibt dies wegen der Gültigkeit des Krull-Schmidt-Theorems die Isomorphie $\left(P^{\prime} \oplus Q\right)_{p} \cong\left(P \oplus Q^{\prime}\right)_{p}$ und folglich

$$
P^{\prime} \oplus Q \cong P \oplus Q^{\prime}
$$

Setzen wir dies wiederum in die Isomorphie

$$
\operatorname{Tor}(M) \oplus P_{p^{s}}^{\prime} \oplus Q_{p^{s}} \cong \operatorname{Tor}\left(M^{\prime}\right) \oplus P_{p^{s}} \oplus Q_{p^{s}}
$$

ein, die wir aus d) und $(+++)$ für $n=p^{s}$ erhalten, so folgt schließlich $\operatorname{Tor}(M)$ $\cong \operatorname{Tor}\left(M^{\prime}\right)$ und damit $\mathrm{c}$ ).

c) $\Rightarrow$ b). Wie oben folgt aus c) die Isomorphie $P^{\prime} \oplus Q \cong P \oplus Q^{\prime}$, mit $(++)$ also $\mathbb{Q}_{p}$ $\otimes M \cong \mathbb{Q}_{p} \otimes M^{\prime}$. 
b) $\Rightarrow$ a). Aus b) und $(++)$ folgt zunächst $\mathbb{Q}_{p} \otimes\left(P^{\prime} \oplus Q\right) \cong \mathbb{Q}_{p} \otimes\left(P \oplus Q^{\prime}\right)$, mit dem Lemma von Swan also $P^{\prime} \oplus Q \cong P \oplus Q^{\prime}$. Andererseits folgt aus der Isomorphie $\operatorname{Tor}(M) \cong \operatorname{Tor}\left(M^{\prime}\right)$ und den zu $(+++)$ dualen Sequenzen mit dem Lemma von Schanuel für jedes $n \in \mathbb{N}$ die Isomorphie

$$
M_{n}^{*} \oplus Q_{n}^{\prime *} \oplus P_{n}^{*}=M_{n}^{\prime *} \oplus Q_{n}^{*} \oplus P_{n}^{\prime *} .
$$

Zusammen ergibt sich die Isomorphie $M_{n}^{*}=M_{n}^{\prime *}$ für jedes $n \in \mathbb{N}$, insbesondere gilt

$$
M / p^{r} M \cong M^{\prime} / p^{r} M^{\prime}
$$

für alle $r \in \mathbb{N}$. Hieraus folgt die Isomorphie $M \cong M^{\prime}$ durch Bildung des projektiven Limes; z.B. mit dem wohlbekannten Satz, daß der projektive Limes nichtleerer endlicher Mengen nicht leer ist, angewandt auf die Mengen Iso $\left(M / p^{r} M, M^{\prime} / p^{r} M^{\prime}\right)$ der Isomorphismen von $M / p^{r} M$ auf $M^{\prime} / p^{r} M^{\prime}$. q.e.d.

Es wird im folgenden nur das Kriterium b) aus Satz 1.2 benötigt. Insbesondere werden uns Moduln des folgenden Typs interessieren.

Definition 1.3. Ein endlich erzeugter $\mathbb{Z}_{p}[G]$-Modul $M$ heißt quasifrei (vom Rang $m$ ), wenn er kohomologisch trivial und $\mathbb{Q}_{p} \otimes M$ ein freier $\mathbb{Q}_{p}[G]$-Modul $\left(\mathbb{Q}_{p} \otimes M \cong \mathbb{Q}_{p}[G]^{m}\right)$ ist.

Satz 1.4. Für einen endlich erzeugten $\mathbb{Z}_{p}[G]-M o d u l M$ sind die folgenden Aussagen äquivalent:

a) $M$ ist quasifrei vom Rang $m$.

b) $M$ ist kohomologisch trivial und enthält einen Untermodul $N \cong \mathbb{Z}_{p}[G]^{m}$ von endlichem Index in $M$.

c) Es gibt eine exakte Sequenz $0 \rightarrow \mathbb{Z}_{p}[G]^{k} \rightarrow \mathbb{Z}_{p}[G]^{l} \rightarrow M \rightarrow 0$ mit $l-k=m$.

Beweis. a) $\Leftrightarrow$ b). Für endlich erzeugte $\mathbb{Z}_{p}[G]$-Moduln $M$ und $N$ gilt die Isomorphie $\mathbb{Q}_{p} \otimes N \cong \mathbb{Q}_{p} \otimes M$ genau dann, wenn es einen $\mathbb{Z}_{p}[G]$-Homomorphismus $f$ : $N \rightarrow M$ mit endlichem Kern und Cokern gibt.

a) $\Rightarrow$ c). Ist für geeignetes $l \in \mathbb{N} f: \mathbb{Z}_{p}[G]^{l} \rightarrow M$ eine Surjektion, so ist $P=\operatorname{Ker} f$ wegen der kohomologischen Trivialität von $M$ ebenfalls kohomologisch trivial, also projektiv wegen $\operatorname{Tor}(P)=0$. Durch Tensorieren mit $\mathbb{Q}_{p}$ folgt $\mathbb{Q}_{p} \otimes P$ $=\mathbb{Q}_{p}[G]^{l-m}$, mit dem Lemma von Swan also $P \cong \mathbb{Z}_{p}[G]^{l-m}$. c) $\Rightarrow$ a) ist klar.

Corollar 1.5. Ist $G$ eine p-Gruppe, so ist jeder endlich erzeugte kohomologisch triviale $\mathbb{Z}_{p}[G]$-Modul quasifrei.

Beweis. Es gibt eine exakte Sequenz $0 \rightarrow P_{1} \rightarrow P_{2} \rightarrow M \rightarrow 0$ mit projektiven Moduln $P_{1}$ und $P_{2}$. Da für eine $p$-Gruppe $G$ der Gruppenring $\mathbb{Z}_{p}[G]$ ein lokaler Ring ist (vgl. [4], $\S 10.5$, Prop. 10), sind $P_{1}$ und $P_{2}$ freie $\mathbb{Z}_{p}[G]$-Moduln (s. etwa [5], Prop. 3.16).

Aus Satz 1.2 folgt, daß quasifreie Moduln $M$ allein durch ihren Rang $m$ (nicht zu verwechseln mit dem $\mathbb{Z}_{p}$-Rang von $M$ ) und ihren Torsionsmodul $\operatorname{Tor}(M)$ bestimmt sind. Die Beziehungen zwischen $M, m$ und $\operatorname{Tor}(M)$ sollen nun weiter untersucht werden. 
Definition 1.6. Für einen endlich erzeugten $\mathbb{Z}_{p}[G]$-Modul $N$ sei der Erzeugendenrang $d=d_{G}(N)$ als die minimale Anzahl von $\mathbb{Z}_{p}[G]$-Erzeugenden von $N$ und der Relationenrang $r=r_{G}(N)$ als der Erzeugendenrang von $\operatorname{Ker} f$ für eine Surjektion $f: \mathbb{Z}_{p}[G]^{d} \rightarrow N$ definiert.

Bemerkung 1.7. a) Ist $g: \mathbb{Z}_{p}[G]^{n} \rightarrow N$ eine weitere Surjektion, $n \geqq d$, so folgt mit dem Lemma von Schanuel

$$
\operatorname{Ker} f \oplus \mathbb{Z}_{p}[G]^{n} \cong \operatorname{Ker} g \oplus \mathbb{Z}_{p}[G]^{d}
$$

und daher $r=r_{G}(N)=d_{G}(\operatorname{Ker} f)=d_{G}(\operatorname{Ker} g)+d-n(v g l$. [5], Lemma 5.8), da $\mathbb{Z}_{p}[G]$ ein semi-lokaler Ring ist. Dies zeigt die Wohldefiniertheit des Relationenranges.

b) Ist $N$ endlich, so gilt $r \geqq d$, wie aus der exakten Sequenz

$$
\mathbb{Z}_{p}[G]^{r \stackrel{\varphi}{\longrightarrow}} \mathbb{Z}_{p}[G]^{d} \rightarrow N \rightarrow 0
$$

z.B. durch Tensorieren mit $\mathbb{Q}_{p}$ folgt. Weiter gilt $r=d$ genau dann, wenn $N$ kohomologisch trivial ist, denn für $r=d$ ist $\varphi$ notwendig injektiv, und umgekehrt ist für kohomologisch triviales $N$ in einer exakten Sequenz

$$
0 \rightarrow K \rightarrow \mathbb{Z}_{p}[G]^{d} \rightarrow N \rightarrow 0
$$

$K$ projektiv, aus der Isomorphie $\mathbb{Q}_{p} \otimes K \cong \mathbb{Q}_{p}[G]^{d}$ folgt daher $K \cong \mathbb{Z}_{p}[G]^{d}$.

Satz 1.8. Ist $M$ quasifrei und (+) $0 \rightarrow \mathbb{Z}_{p}[G]^{k} \rightarrow \mathbb{Z}_{p}[G]^{l} \stackrel{\pi}{\longrightarrow} M \rightarrow 0$ exakt, so gelten mit $d=d_{G}\left(\operatorname{Tor}(M)^{*}\right)$ und $r=r_{G}\left(\operatorname{Tor}(M)^{*}\right)$ die Ungleichungen $k \geqq d, l \geqq r$ und $l-k \geqq r-d$.

Beweis. Aus $(+)$ folgt die exakte Sequenz

$$
\begin{aligned}
0 & \rightarrow \operatorname{Hom}\left(M, \mathbb{Z}_{p}\right) \rightarrow \operatorname{Hom}\left(\mathbb{Z}_{p}[G]^{l}, \mathbb{Z}_{p}\right) \\
& \left.\rightarrow \operatorname{Hom}\left(\mathbb{Z}_{p}[G]^{k}, \mathbb{Z}_{p}\right) \stackrel{\delta}{\longrightarrow} \operatorname{Tor}(M)\right)^{*} \rightarrow 0
\end{aligned}
$$

wegen der kanonischen Isomorphie $\operatorname{Ext}_{\mathbf{z}_{p}}^{1}\left(M, \mathbb{Z}_{p}\right) \cong \operatorname{Tor}_{1}^{\mathbb{Z}_{p}}\left(M, \mathbb{Q}_{p} / \mathbb{Z}_{p}\right)^{*}=\operatorname{Tor}(M)^{*}$ ([2], VI, Prop.5.4 u. $\left.\left(4^{\prime}\right)\right)$ und $\operatorname{Ext}_{\mathbb{Z}_{p}}^{1}\left(\mathbb{Z}_{p}[G]^{l}, \mathbb{Z}_{p}\right)=0$. Explizit definiere etwa $\delta(f) \in \operatorname{Tor}(M)^{*}$ durch $\delta(f)(x)=f\left(p^{m} y\right) p^{-m}+\mathbb{Z}_{p} \in \mathbb{Q}_{p} / \mathbb{Z}_{p}$ für $x \in \operatorname{Tor}(M)$ mit $p^{m} x$ $=0, \pi(y)=x$. Da $\operatorname{Hom}\left(\mathbb{Z}_{p}[G]^{n}, \mathbb{Z}_{p}\right)$ wieder ein freier $\mathbb{Z}_{p}[G]$-Modul vom Rang $n$ ist, erhalten wir eine exakte Sequenz

$$
\mathbb{Z}_{p}[G]^{l} \rightarrow \mathbb{Z}_{p}[G]^{k} \stackrel{\delta}{\longrightarrow} \operatorname{Tor}(M)^{*} \rightarrow 0 .
$$

Daraus folgt sofort $k \geqq d$, sowie $l \geqq d_{G}(\operatorname{Ker} \delta)=r+k-d(\mathrm{vgl}$. Bem. 1.7a)). Aus beiden Ungleichungen folgt schließlich auch $l \geqq r$.

Aus dem obigen Satz folgt insbesondere, daß für einen endlichen $\mathbb{Z}_{p}[G]$ Modul $N$ die Ränge der quasifreien Moduln $M$ mit $\operatorname{Tor}(M) \cong N$ durch die Zahl $r_{G}\left(N^{*}\right)-d_{G}\left(N^{*}\right)$ nach unten beschränkt sind. Der folgende Satz zeigt, daß es auch immer einen quasifreien Modul mit diesem minimalen Rang gibt und wie allgemein quasifreie Moduln aus ihren Torsionsmoduln konstruiert werden können. Insbesondere gibt es zu jedem endlichen $\mathbb{Z}_{p}[G]$-Modul $N$ einen kohomologisch trivialen Modul $M$ (sogar einen quasifreien) mit $\operatorname{Tor}(M) \cong N$. 
Satz 1.9. Sei $N$ ein endlicher $\mathbb{Z}_{p}[G]$-Modul und

$$
P \stackrel{f}{\longrightarrow} Q \rightarrow N^{*} \rightarrow 0
$$

exakt mit $P=\mathbb{Z}_{p}[G]^{l}$ und $Q=\mathbb{Z}_{p}[G]^{k}$. Dann ist die von $f$ induzierte Abbildung $f^{+}: Q^{+} \rightarrow P^{+}$injektiv und $M=$ Coker $f^{+}$ist der quasifreie $\mathbb{Z}_{p}[G]$-Modul vom Rang $l-k$ mit Torsionsmodul $N$.

Beweis. Die Injektivität von $f^{+}$folgt wegen $\operatorname{Hom}\left(N^{*}, \mathbb{Z}_{p}\right)=0$ aus der Linksexaktheit des Hom-Funktors. $M=$ Coker $f^{+}$ist quasifrei vom Rang $l-k$, da $P^{+}$ bzw. $Q^{+}$freie $\mathbb{Z}_{p}[G]$-Moduln vom Rang $l$ bzw. $k$ sind. Durch erneute Anwendung des Hom-Funktors erhalten wir die exakte Sequenz

$$
0 \rightarrow M^{+} \rightarrow P^{++} \stackrel{s^{++}}{\longrightarrow} Q^{++} \rightarrow \operatorname{Tor}(M)^{*} \rightarrow 0,
$$

wieder wegen $\operatorname{Ext}_{\mathbb{Z}_{p}}^{1}\left(M, \mathbb{Z}_{p}\right) \cong \operatorname{Tor}(M)^{*}$, und in dem kommutativen Diagramm

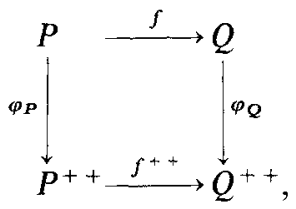

sind die kanonischen Abbildungen $\varphi_{P}$ und $\varphi_{Q}$ wegen der Torsionsfreiheit von $P$ und $Q$ Isomorphismen. Daraus folgt $N^{*}=\operatorname{Coker} f \cong \operatorname{Coker} f^{++} \cong \operatorname{Tor}(M)^{*}$, also $N \cong \operatorname{Tor}(M)$. q.e.d.

Corollar 1.10. Sei $N$ ein endlicher $\mathbb{Z}_{p}[G]-M o d u l, d=d_{G}\left(N^{*}\right)$ und $r=r_{G}\left(N^{*}\right)$, damn existiert ein quasifreier Modul $M_{0}(N)$ mit Torsionsmodul $N$ vom minimalen Rang $r-d$; für ihn gibt es eine exakte Sequenz

$$
0 \rightarrow \mathbb{Z}_{p}[G]^{d} \rightarrow \mathbb{Z}_{p}[G]^{r} \rightarrow M_{0}(N) \rightarrow 0 .
$$

Ist $M$ quasifrei vom Rang $m=r-d+j, j \geqq 0$, mit $\operatorname{Tor}(M) \cong N$, so gilt die Isomorphie $M \cong M_{0}(N) \oplus \mathbb{Z}_{p}[G]^{j}$ und es gibt eine exakte Sequenz

$$
0 \rightarrow \mathbb{Z}_{p}[G]^{d} \rightarrow \mathbb{Z}_{p}[G]^{m+d} \rightarrow M \rightarrow 0 .
$$

Beweis. Die Konstruktion von 1.9 liefert aus einer exakten Sequenz

$$
\mathbb{Z}_{p}[G]^{l} \stackrel{f}{\longrightarrow} \mathbb{Z}_{p}[G]^{k} \rightarrow N^{*} \rightarrow 0
$$

eine exakte Sequenz

$$
0 \rightarrow \mathbb{Z}_{p}[G]^{k} \stackrel{f^{+}}{\longrightarrow} \mathbb{Z}_{p}[G]^{l} \rightarrow M \rightarrow 0
$$

mit $\operatorname{Tor}(M) \cong N$. Für $k=d$ und $l=r$ ergibt sich die erste Aussage. Die lsomorphie $M=M_{0}(N) \oplus \mathbb{Z}_{p}[G]^{j}$ folgt aus Satz 1.2, und aus der Sequenz für $M_{0}(N)$ erhält man trivialerweise eine exakte Sequenz

$$
0 \rightarrow \mathbb{Z}_{p}[G]^{d} \rightarrow \mathbb{Z}_{p}[G]^{r+j} \rightarrow M_{0}(N) \oplus \mathbb{Z}_{p}[G]^{j} \rightarrow 0,
$$

in der $r+j=m+d$ gilt. 
Die obigen Betrachtungen führen zu einer allgemeinen Strukturaussage für kohomologisch triviale $\mathbb{Z}_{p}[G]$-Moduln. Jeder endlich erzeugte $\mathbb{Z}_{p}[G]$-Modul $M$ läßt sich aufspalten in $M=\tilde{M} \oplus P$, wobei $P$ projektiv ist und $\tilde{M}$ keine projektiven Summanden mehr besitzt. Der Modul $\tilde{M}$ ist bis auf Isomorphie eindeutig bestimmt (vgl. hierzu [16], Paragraph 6).

Satz 1.11. Sei $M$ ein endlich erzeugter, kohomologisch trivialer $\mathbb{Z}_{p}[G]$-Modul und $N=\operatorname{Tor}(M)$, dann gilt $\tilde{M} \cong \widetilde{M_{0}(N)}$. Anders ausgedrückt: Alle endlich erzeugten, kohomologisch trivialen $\mathbb{Z}_{p}[G]$-Moduln $M$ mit $\operatorname{Tor}(M) \cong N$ haben die Gestalt $M$ $\cong \widehat{M_{0}(N)} \oplus P$ mit projektivem $P$.

Beweis. Sei $0 \rightarrow Q \rightarrow \mathbb{Z}_{p}[G]^{k} \rightarrow M \rightarrow 0$ exakt mit projektivem $Q$, dann ist offenbar $M \oplus Q$ quasifrei mit $\operatorname{Tor}(M \oplus Q)=\operatorname{Tor}(M)=N$, also isomorph zu $M_{0}(N)$ $\oplus \mathbb{Z}_{p}[G]^{j}$ für ein geeignetes $j \geqq 0$. Aus der Zerlegung

$$
\tilde{M} \oplus P \oplus Q=\widetilde{M_{0}(N)} \oplus P^{\prime} \oplus \mathbb{Z}_{p}[G]^{j}
$$

folgt wegen der Eindeutigkeit $\tilde{M}=\widetilde{M_{0}(N)}$. q.e.d.

Bemerkung 1.12. a) Ist $G$ eine $p$-Gruppe, so gilt $\widetilde{M_{0}(N)}=M_{0}(N)$, denn in einer Zerlegung $M_{0}(N)=\widehat{M_{0}(N)} \oplus P$ ist $P$ frei; aus der Minimalität von $M_{0}(N)$ folgt $P=0$.

b) Ist $N$ unzerlegbar, so auch $\widetilde{M_{0}(N)}$, denn in einer Zerlegung $\widetilde{M_{0}(N)}=A$ $\oplus B$ wäre einer der Faktoren torsionsfrei, also projektiv wegen der kohomologischen Trivialität von $\widehat{M_{0}(N)}$.

c) Ist $N=\oplus N_{i}$ mit unzerlegbaren $N_{i}$, so ist $\widehat{M_{0}(N)} \cong \oplus \widetilde{M_{0}\left(N_{i}\right)}$, denn $\oplus \widetilde{M_{0}\left(N_{i}\right)}$ ist kohomologisch trivial mit Torsionsmodul $N$ und besitzt keinen projektiven Summanden, da die $\widehat{M_{0}\left(N_{i}\right)}$ unzerlegbar und nicht projektiv sind. Dadurch ist aber gerade $\widetilde{M_{0}(N)}$ charakterisiert.

\section{§ 2. Zahm-verzweigte Erweiterungen lokaler Körper}

Ist $k$ ein lokaler Körper, dessen Restklassenkörper die Charakteristik $p$ hat, so ist die Gruppe $U_{k}^{1}$ der Einseinheiten von $k$ als abelsche pro-p-Gruppe ein $\mathbb{Z}_{p^{-}}$ Modul. Für eine endliche galoissche Erweiterung $K / k$ wird $U_{k}^{1}$ durch die stetige Operation der Automorphismen der Galoisgruppe $G$ zu einem $\mathbb{Z}_{p}[G]$ Modul. In diesem Paragraphen werden nur p-adische Zahlkörper behandelt; die Potenzreihenkörper werden am Ende von $\$ 3$ betrachtet.

Satz 2.1. Sei Char $(k)=0$, also $k$ eine endliche Erweiterung des Körpers $\mathbb{Q}_{p}$ der $p$-adischen Zahlen. Ist $K / k$ eine endliche, zahm-verzweigte, galoissche Erweiterung mit Galoisgruppe $G$ sowie $\mu_{K}$ die Gruppe der in $K$ enthaltenen Einheitswurzeln von p-Potenz-Ordnung, dann gilt mit $n=\left[k: \mathbb{Q}_{p}\right]$

a) $U_{K}^{1}$ ist der (eindeutig bestimmte) quasifreie $\mathbb{Z}_{p}[G]$-Modul vom Rang $n$ mit Torsionsmodul $\mu_{K}$. 
b) Es gibt eine exakte Sequenz $0 \rightarrow \mathbb{Z}_{p}[G] \rightarrow \mathbb{Z}_{p}[G]^{n+1} \rightarrow U_{K}^{1} \rightarrow 1$.

c) Ist $K$ regulär, d.h., enthält $K$ keine primitive p-te Einheitswurzel, so gilt $U_{K}^{1} \cong \mathbb{Z}_{p}[G]^{n}$.

Beweis. a) Für jede galoissche Erweiterung $K / k$ mit Galoisgruppe $G$ enthält $U_{K}^{1}$ einen freien $\mathbb{Z}_{p}[G]$-Modul vom Rang $n$ und endlichem Index in $U_{K}^{1}$, d.h., es gilt $\mathbb{Q}_{p} \otimes U_{K}^{1} \cong \mathbb{Q}_{p}[G]^{n}$. Dies ist wohlbekannt und folgt mit Hilfe der $\mathfrak{p}$-adischen Exponentialfunktion aus der Existenz einer Normalbasis (vgl. Gilbarg [3]). Für eine zahm-verzweigte Erweiterung ist zusätzlich $U_{K}^{1}$ kohomologisch trivial; dies folgt z.B. aus der kohomologischen Trivialität von $U_{K}^{1}$ unter einer $p$-Sylowgruppe $G_{p}$, die einer unverzweigten Erweiterung entspricht (vgl. hierzu [14], XII, $\S 3)$. Schließlich gilt gerade $\operatorname{Tor}\left(U_{K}^{1}\right)=\mu_{K}$.

b) Folgt aus 1.10 wegen $d_{G}\left(\mu_{K}^{*}\right)=1$.

c) Ist $K$ regulär, so ist $U_{K}^{1}$ torsionsfrei, wegen der kohomologischen Trivialität also projektiv. Aus der Isomorphie $\mathbb{Q}_{p} \otimes U_{K}^{1} \cong \mathbb{Q}_{p} \otimes \mathbb{Z}_{p}[G]^{n}$ folgt dann mit dem Lemma von Swan sofort $U_{K}^{1} \cong \mathbb{Z}_{p}[G]^{n}$. q.e.d.

Mit Hilfe von Satz 1.9 soll nun die Sequenz aus $2.1 \mathrm{~b}$ ) explizit angegeben und damit $U_{K}^{1}$ durch $\mathbb{Z}_{p}[G]$-Erzeugende und -Relationen beschrieben werden.

Die betrachtete Gruppe $G$ besitzt nach Hasse [6], $\$ 16$ Erzeugende $\sigma$ und $\tau$, die der Relation

$$
\sigma \tau \sigma^{-1}=\tau^{q}
$$

genügen, wobei $q=p^{f_{0}}$ die Anzahl der Elemente des Restklassenkörpers von $k$ ist. Wir beschreiben allgemein für eine derartige Gruppe quasifreie $\mathbb{Z}_{p}[G]$ Moduln mit zyklischem Torsionsmodul $N$. Ist $N$ als abelsche Gruppe isomorph zu $\mathbb{Z} / p^{s} \mathbb{Z}$, so wird die Operation von $G$ auf $N$ gegeben durch einen Charakter

$$
\alpha: G \rightarrow\left(\mathbb{Z} / p^{s} \mathbb{Z}\right)^{\times}
$$

von $G$ in die Einheitengruppe von $\mathbb{Z} / p^{s} \mathbb{Z}$ mit $\rho y=\alpha(\rho) \cdot y$ für alle $\rho \in G$ und $y \in N$. Es sei $g \in \mathbb{Z}_{p}$ eine beliebige Liftung von $\alpha(\sigma), h$ eine von $\alpha(\tau), y^{*}$ ein Erzeugendes von $N^{*}$ und

$$
\varphi: \mathbb{Z}_{p}[G] \rightarrow N^{*}
$$

der surjektive $\mathbb{Z}_{p}[G]$-Homomorphismus mit $\varphi(1)=y^{*}$. Dann wird der Kern von $\varphi$ als $\mathbb{Z}_{p}[G]$-Modul offenbar von den drei Elementen

$$
\sigma^{-1}-g, \tau^{-1}-h \text { und } p^{s}
$$

erzeugt ( $G$ operiert auf $N^{*}$ vermöge $\alpha^{-1}$ ). Bezeichnet ${ }^{0}$ die Anti-Involution auf dem Gruppenring $\mathbb{Z}_{p}[G]$, die durch $\left(\sum_{\rho \in G} c_{\rho} \rho\right)^{0}=\sum_{\rho \in G} c_{\rho} \rho^{-1}$ gegeben wird, $e$ die Ordnung von $\tau$, die notwendigerweise prim $\mathrm{zu} p$ ist, und ist $\lambda \in \mathbb{Z}_{p}[G]$ eine beliebige Liftung des Elementes

$$
\bar{\lambda}=\frac{1}{e} \sum_{i=0}^{e-1} \tau^{i} \alpha(\tau)^{-i} \in \mathbb{Z} / p^{s} \mathbb{Z}[G]
$$


so wird $\operatorname{Ker} \varphi$ auch erzeugt durch die zwei Elemente

$$
\mu=\sigma^{-1}-g \lambda^{0} \quad v=-p^{s} .
$$

(Es gilt $\tau \bar{\lambda}=\alpha(\tau) \bar{\lambda}$ und $\sigma \bar{\lambda} \sigma^{-1}=\bar{\lambda}$ wegen $\sigma \tau \sigma^{-1}=\tau^{p_{0}}$ und $\alpha(\tau)^{p-1}=1$. Daraus folgt $\left(\tau^{-1}-h\right) \sigma \mu \equiv\left(\tau^{-1}-h\right)-g\left(\tau^{-1}-h\right) \lambda^{0} \sigma \equiv \tau^{-1}-h \bmod p^{s}$. Setzt man $A$ $=\mathbb{Z}_{p}[G] \mu+\mathbb{Z}_{p}[G] \nu \subseteq \operatorname{Ker} \varphi$, so gilt also $\left(\tau^{-1}-h\right) \in A$ bzw. $\tau^{-1} \equiv h \bmod A$. Hieraus folgt wiederum $\lambda^{0} \equiv \frac{1}{e} \sum_{i=0}^{e-1} \tau^{-i} h^{-i} \equiv \frac{1}{e} \cdot e \equiv 1 \bmod A$ bzw. $\sigma^{-1}-g=\mu-g\left(\lambda^{0}\right.$ $-1) \equiv 0 \bmod A$. Die Erzeugenden (1) von $\operatorname{Ker} \varphi$ liegen also alle in $A$.)

Wir erhalten daher eine exakte Sequenz

$$
\mathbb{Z}_{p}[G]^{2} \stackrel{\psi}{\longrightarrow} \mathbb{Z}_{p}[G] \stackrel{\varphi}{\longrightarrow} N^{*} \rightarrow 0,
$$

indem wir $\psi(a)=\mu$ und $\psi(b)=v$ für eine Basis $\{a, b\}$ von $\mathbb{Z}_{p}[G]^{2}$ setzen. Nach Satz 1.9 liefert die Bildung der $\mathbb{Z}_{p}$-Duale eine exakte Sequenz

$$
0 \rightarrow \mathbb{Z}_{p}[G] 1^{+} \stackrel{\psi^{+}}{\longrightarrow} \mathbb{Z}_{p}[G] a^{+} \oplus \mathbb{Z}_{p}[G] b^{+} \rightarrow M_{1}(N) \rightarrow 0
$$

für den quasifreien $\mathbb{Z}_{p}[G]$-Modul $M_{1}(N)$ vom Rang 1 mit Torsionsmodul $N$. Hierbei sei $1^{+}$das $\mathbb{Z}_{p}[G]$-Basiselement von $\mathbb{Z}_{p}[G]^{+}$mit

$$
1^{+}\left(\sum_{\rho \in G} c_{\rho} \rho\right)=c_{1}
$$

und $\left\{a^{+}, b^{+}\right\}$die $\mathbb{Z}_{p}[G]$-Basis von $\left(\mathbb{Z}_{p}[G] a \oplus \mathbb{Z}_{p}[G] b\right)^{+}$mit

$$
\begin{aligned}
& a^{+}(\gamma)=a_{1} \\
& b^{+}(\gamma)=b_{1}
\end{aligned} \quad \text { für } \gamma=\left(\sum_{\rho \in G} a_{\rho} \rho\right) a+\left(\sum_{\rho \in G} b_{\rho} \rho\right) b
$$

Eine leichte Rechnung zeigt dann

$$
\psi^{+}\left(1^{+}\right)=(\sigma-g \lambda) a^{+}-p^{s} b^{+} .
$$

Nach Satz 2.1 gilt für $N \cong \mu_{K}$ die Isomorphie $U_{K}^{1} \cong M_{1}(N) \oplus \mathbb{Z}_{p}[G]^{n-1}$ und damit

Satz 2.2. Sei $k$ vom Grad $n$ über $\mathbb{Q}_{p}, K / k$ eine (endliche) zahm-verzweigte, normale Erweiterung und $\mu_{K}$ die Gruppe der p-Potenz-Einheitswurzeln in $K$. Sind $\sigma$ und $\tau$ Erzeugende der Galoisgruppe $G$, die der Relation $\sigma \tau \sigma^{-1}=\tau^{q}$ genügen ( $q$ die Mächtigkeit des Restklassenkörpers von $k$ ), ist

$$
\alpha: G \rightarrow\left(\mathbb{Z} / p^{s} \mathbb{Z}\right)^{\times}, \quad p^{s}=\left(\mu_{K}: 1\right),
$$

der Charakter mit $\zeta^{\rho}=\zeta^{\alpha(\rho)}$ für alle $\rho \in G$ und $\zeta \in \mu_{K}, g \in \mathbb{Z}_{p}$ eine Liftung von $\alpha(\sigma)$ und $\lambda \in \mathbb{Z}_{p}[G]$ eine Liftung von

$$
\bar{\lambda}=\frac{1}{e} \sum_{i=0}^{e-1} \tau^{i} \alpha(\tau)^{-i}, \quad \text { e die Ordnung von } \tau,
$$

(z.B. $\lambda=\frac{1}{e} \sum_{i=0}^{e-1} \tau^{i} h^{-i}$ mit einer Liftung $h$ von $\left.\alpha(\tau)\right)$, so besitzt $U_{K}^{1} \mathbb{Z}_{p}[G]$-Erzeu- 
gende $\eta_{0}, \ldots, \eta_{n}$ mit der definierenden $\mathbb{Z}_{p}[G]$-Relation

$$
\eta_{0}^{\sigma}=\eta_{0}^{g \lambda} \cdot \eta_{1}^{p^{s}}
$$

Weiter ist die Sequenz

$$
0 \rightarrow \mathbb{Z}_{p}[G] \rightarrow \oplus_{i=0}^{n} \mathbb{Z}_{p}[G] b_{i} \rightarrow U_{K}^{1} \rightarrow 1
$$

mit $1 \mapsto(\sigma-g \lambda) b_{0}-p^{s} b_{1}, b_{i} \mapsto \eta_{i}$ exakt.

Bemerkung 2.3. Mit der obigen Methode kann man noch andere Darstellungen von $U_{\mathrm{K}}^{1}$ erhalten; dafür sei die Liftung $h$ von $\alpha(\tau)$ als $(p-1)$-te Einheitswurzel in $\mathbb{Z}_{p}$ gewählt.

a) $\operatorname{Ker} \varphi$ wird auch durch $\mu^{\prime}=\sigma^{-1}-g$ und $v^{\prime}=\tau^{-1}-h+p^{s}$ erzeugt; daher gibt es Erzeugende $\eta_{0}, \eta_{1}, \ldots, \eta_{n}$ von $U_{\mathbf{K}}^{1}$ mit der definierenden Relation

$$
\eta_{0}^{\sigma-g} \eta_{1}^{\tau-\boldsymbol{h}+\boldsymbol{p}^{s}}=1 .
$$

Da in ihr die Summe $\lambda$ nicht auftaucht, ist sie einfacher und erscheint auf den ersten Blick günstiger als die oben beschriebene. Diese hat sich aber für weitere Untersuchungen über die absolute Galoisgruppe von $k$ (s. [9]) als am geeignetsten erwiesen.

b) Erzeugt man $\operatorname{Ker} \varphi$ durch die Elemente $\sigma^{-1}-g \lambda^{0}$ und $-p^{s} \lambda^{0}$, mit $\lambda$ $=\frac{1}{e} \sum_{i=0}^{e-1} \tau^{i} h^{-i}$, so erhält man entsprechend eine Relation

$$
\eta_{0}^{\sigma-g \lambda} \eta_{1}^{-p^{s} \lambda}=1,
$$

die aufgrund der Beziehungen $(\tau-h) \lambda=0$ und $\sigma \lambda=\lambda \sigma$ äquivalent $z u$ den beiden Relationen

$$
\eta_{0}^{\tau-h}=1 \quad \eta_{0}^{\sigma-g}=\eta_{1}^{p^{s} \lambda}
$$

ist. Dies ist die Darstellung von Iwasawa [7].

\section{§3. Die absolute Galoisgruppe lokaler Körper}

Wir betrachten im folgenden einen lokalen Körper $k$ mit Restklassenkörper $\mathbb{F}_{q}$ der Charakteristik $p$ und eine $p$-abgeschlossene Erweiterung $L$ von $k$, d.h., eine galoissche Erweiterung, die keiner (galoisschen) $p$-Erweiterung mehr fähig ist. Sei $T$ die maximale zahm-verzweigte Erweiterung von $k$ in $L$, weiter $G_{k}$ $=G(L / k), \mathscr{G}=G(T / k)$ sowie $V_{k}=G(L / T)$ die Verzweigungsgruppe von $L / k$. Für einen separablen Abschluß $L$ von $k$ ist $G_{k}$ also die absolute Galoisgruppe von $k$ und $\mathscr{G}$ nach Iwasawa [7] die pro-endliche Gruppe mit zwei Erzeugenden $\sigma$ und $\tau$ und der definierenden Relation

$$
\sigma \tau \sigma^{-1}=\tau^{q}
$$

( $\tau$ erzeugt die Trägheitsgruppe $\mathscr{G}_{0} \cong \hat{\mathbb{Z}} / \mathbb{Z}_{p} \cong \prod_{\ell \neq p} \mathbb{Z}_{\ell}$, und $\sigma$ liftet den Frobenius- 
automorphismus, $\mathscr{G} / \mathscr{G}_{0} \cong \hat{\mathbb{Z}}$ ). Im allgemeinen Fall handelt es sich um Faktorgruppen hiervon.

Satz 3.1. Es gilt $c d_{p}(\mathscr{G})=1, V_{k}$ ist eine freie pro-p-Gruppe, und die exakte Sequenz $1 \rightarrow V_{k} \rightarrow G_{k} \rightarrow \mathscr{G} \rightarrow 1$ zerfällt.

Beweis. Da $T$ die maximale unverzweigte $p$-Erweiterung enthält, ist der $p$ Anteil der Brauergruppe null für $T$ und alle Oberkörper; folglich hat die absolute Galoisgruppe von $T$ und damit auch ihre maximale pro- $p$-Faktorgruppe $V_{k}$ die kohomologische $p$-Dimension eins. Weiter ist jede $p$-Sylowgruppe von $\mathscr{G}$ isomorph zu $\mathbb{Z}_{p}$, also $c d_{p}(\mathscr{G})=1$, woraus wiederum das Zerfallen der Sequenz folgt (s. [15], I, Prop. 16).

Für eine pro-endliche Gruppe $H$ sei $[H, H]$ die topologische Kommutatorgruppe, $H^{\mathrm{ab}}=H /[H, H]$ die Faktorkommutatorgruppe und $H(p)$ die maximale pro- $p$-Faktorgruppe.

Ist $\mathscr{H}$ ein offener Normalteiler von $\mathscr{G}$ und $K=T^{\mathscr{H}}$ die entsprechende zahmverzweigte Erweiterung von $k$, so induziert das universelle Normrestsymbol für $L / K$ einen Isomorphismus

$$
\omega_{K}: U_{K}^{1} \stackrel{\sim}{\longrightarrow} V_{k}\left[G_{K}, G_{K}\right] /\left[G_{K}, G_{K}\right] \text { mit } G_{K}=G(L / K),
$$

da $L p$-abgeschlossen (Injektivität) und die rechts stehende Gruppe die Verzweigungsgruppe von $G_{K}^{\text {ab }}$ ist (Surjektivität). Der projektive Limes der Gruppen auf der rechten Seite, gebildet über alle $K \subseteq T$ mit den kanonischen Surjektionen, ist gerade $V_{k}^{\mathrm{ab}}$; dies liefert einen topologischen Isomorphismus

$$
\phi: V_{k}^{\text {ab }} \underset{\substack{\text { K/kendl. gal. } \\ \text { zahm-verzw. }}}{\sim} U_{K}^{1},
$$

wobei die Abbildungen zwischen den Einseinheitengruppen nach der Klassenkörpertheorie die Normen sind.

$V_{k}^{\mathrm{ab}}$ ist ein Modul über dem komplettierten Gruppenring

$$
\mathbb{Z}_{p} \llbracket \mathscr{G} \rrbracket=\lim _{\substack{\mathscr{\mathscr { T }} \triangleleft \mathscr{G} \\ \text { offen }}} \mathbb{Z}[\mathscr{G} / \mathscr{H}]
$$

s. Brumer [1]; dabei wird die Operation von $\mathscr{G}$ durch die inneren Automorphismen von $G_{k}$ induziert. Faßt man auch die Einseinheitengruppen $U_{\mathbf{K}}^{1}$ durch die kanonische Projektion von $\mathbb{Z}_{p} \llbracket \mathscr{G} \rrbracket$ auf $\mathbb{Z}_{p}[\mathscr{G} / \mathscr{H}]$ als $\mathbb{Z}_{p} \llbracket \mathscr{G} \rrbracket$-Moduln auf, so ist $\phi$ ein $\mathbb{Z}_{p} \llbracket \mathscr{G} \rrbracket$-Isomorphismus.

\section{A) p-adische Zahlkörper}

Satz 3.2. Ist $k$ vom Grad $n$ über $\mathbb{Q}_{p}$, so gibt es eine exakte Sequenz von $\mathbb{Z}_{p} \llbracket \mathscr{G} \rrbracket-$ Moduln

$$
0 \rightarrow \mathbb{Z}_{p} \llbracket \mathscr{G} \rrbracket \rightarrow \mathbb{Z}_{p} \llbracket \mathscr{G} \rrbracket^{n+1} \rightarrow V_{k}^{\mathrm{ab}} \rightarrow 1
$$


a) Enthält T keine primitive p-te Einheitswurzel, so gilt die Isomorphie

$$
V_{k}^{\mathrm{ab}} \cong \mathbb{Z}_{p} \llbracket \mathscr{G} \rrbracket^{n}
$$

b) Die Gruppe $\mu_{T}$ der in $T$ enthaltenen Einheitswurzeln von p-Potenz-Ordnung habe die Ordnung $p^{s}, s \geqq 1$, und

$$
\alpha: \mathscr{G} \rightarrow\left(\mathbb{Z} / p^{s} \mathbb{Z}\right)^{\times}
$$

sei der Charakter mit $\zeta^{\rho}=\zeta^{\alpha(\rho)}$ für alle $\rho \in \mathscr{G}$ und $\zeta \in \mu_{T}$. Weiter seien $\sigma$ und $\tau$ topologische Erzeugende von $\mathscr{G}$, die der Relation $\sigma \tau \sigma^{-1}=\tau^{q}$ genügen, $g \in \mathbb{Z}_{p}$ eine Liftung von $\alpha(\sigma)$ und $\lambda$ ein Element aus $\mathbb{Z}_{p} \llbracket \mathscr{G} \rrbracket$, das für alle offenen Normalteiler $\mathscr{H} \subseteq \operatorname{Ker} \alpha$ von $\mathscr{G}$ bei der Projektion auf $\mathbb{Z} / p^{s} \mathbb{Z}[\mathscr{G} / \mathscr{H}]$ jeweils auf

$$
\bar{\lambda}_{\mathscr{H}}=\frac{1}{e_{\mathscr{H}}} \sum_{i=0}^{e_{\mathscr{H}}-1} \bar{\tau}^{i} \alpha(\tau)^{-i}, \quad e_{\mathscr{H}} \text { die Ordnung von } \bar{\tau}=\tau \mathscr{H}
$$

abgebildet wird. Dann gibt es $\mathbb{Z}_{p} \llbracket \mathscr{G} \rrbracket$-Erzeugende $\bar{x}_{0}, \bar{x}_{1}, \ldots, \bar{x}_{n}$ von $V_{k}^{\mathrm{ab}}$ mit der definierenden Relation

$$
\bar{x}_{0}^{\sigma}=\bar{x}_{0}^{g \lambda} \cdot \bar{x}_{1}^{p^{s}}
$$

(Die Gruppe $\mu_{T}$ ist aus Verzweigungsgründen endlich. Ist $\beta: \mathscr{G} \rightarrow \mathbb{Z}_{p}^{\times}$eine Liftung (als Abbildung) von $\alpha$, deren Stabilisator einen offenen Normalteiler $\mathscr{H}^{\prime}$ von $\mathscr{G}$ enthält, so kann z.B. ein $\lambda$ mit der verlangten Eigenschaft durch die verträgliche Familie $\lambda_{\mathscr{H}}=\frac{1}{e_{\mathscr{H}}} \sum_{i=0}^{e_{\mathscr{H}}-1} \bar{\tau}^{i} \beta\left(\tau^{i}\right)^{-1} \in \mathbb{Z}_{p}[\mathscr{G} / \mathscr{H}]$ für $\mathscr{H} \subseteq \mathscr{H}^{\prime}$ definiert werden. Insbesondere kann man die Liftung $\beta\left(\tau^{i}\right)=h^{i}$ mit einer $(p-1)$-ten Einheitswurzel $h \in \mathbb{Z}_{p}$ wählen.)

Beweis. Für jeden offenen Normalteiler $\mathscr{H}$ von $\mathscr{G}$ und $K=T^{\mathscr{H}}$ sei $A_{\mathscr{H}}$ die Menge der $(n+1)$-Tupel $\left(z_{0}, \ldots, z_{n}\right) \in\left(V_{k}^{\mathrm{ab}}\right)^{n+1}$, für die die Bilder $\eta_{i} \operatorname{der} z_{i}$ in $V_{k} /\left[G_{K}, G_{K}\right] \cong U_{K}^{1}$ diese Gruppe als $\mathbb{Z}_{p}[\mathscr{G} / \mathscr{H}]$-Modul erzeugen, wobei die Relation

$$
\eta_{0}^{\sigma}=\eta_{0}^{g \lambda} \cdot \eta_{1}^{p^{s}}
$$

gilt. Dann sind die $A_{\mathscr{H}}$ abgeschlossen und nach Satz 2.2 für $\mathscr{H} \subseteq \mathrm{Ker} \alpha$ bzw. $\mu_{T} \subseteq K$ nicht leer; weiter gilt $A_{\mathscr{H}} \subseteq A_{\mathscr{H}}$ für $\mathscr{H}^{\prime} \subseteq \mathscr{H} \subseteq \mathrm{Ker} \alpha$. Wegen der Kompaktheit von $\left(V_{k}^{\mathrm{ab}}\right)^{n+1}$ ist daher der Durchschnitt aller $A_{\mathscr{H}}$ für $\mathscr{H} \subset \operatorname{Ker} \alpha$ nicht leer. Wählen wir ein Element $\left(\bar{x}_{0}, \ldots, \bar{x}_{n}\right)$ daraus, so wird $V_{k}^{\text {ab }}$ als $\mathbb{Z}_{p} \llbracket \mathscr{G} \rrbracket-$ Modul von $\bar{x}_{0}, \ldots, \bar{x}_{n}$ erzeugt, und es gilt die Relation $(+)$. Dies liefert eine Sequenz

$$
\begin{gathered}
0 \rightarrow \mathbb{Z}_{p} \llbracket \mathscr{G} \rrbracket \rightarrow \underset{i=0}{\oplus} \mathbb{Z}_{p} \llbracket \mathscr{G} \rrbracket a_{i} \rightarrow V_{k}^{\mathrm{ab}} \rightarrow 1 \\
1 \mapsto(\sigma-g \lambda) a_{0}-p^{s} a_{1} ; \quad a_{i} \rightarrow \bar{x}_{i},
\end{gathered}
$$

die exakt ist als projektiver Limes der (nach 2.2) exakten Sequenzen

$$
0 \rightarrow \mathbb{Z}_{p}[\mathscr{G} / \mathscr{H}] \rightarrow \bigoplus_{i=0}^{n} \mathbb{Z}_{p}[\mathscr{G} / \mathscr{H}] a_{i} \rightarrow U_{K}^{1} \rightarrow 1
$$

mit den entsprechenden Abbildungen.

a) folgt mit den gleichen Schlüssen aus $2.1 \mathrm{c}$ ). 
Satz 3.2 gibt nicht nur eine Beschreibung von $G_{k} /\left[V_{k}, V_{k}\right]$, sondern gestattet auch Aussagen über die Struktur der Gruppe $G_{k}$ selbst, insbesondere über die Anzahl von Erzeugenden und Relationen. Als erstes ergibt sich aus 3.2 und dem folgenden Lemma, daß $V_{k}$ als Normalteiler in $G_{k}$ von $n+1$ Elementen erzeugt wird, woraus offenbar folgt, daß $G_{k}$ von $n+3$ Elementen erzeugt wird.

Lemma 3.3. Sei $1 \rightarrow H \rightarrow E \rightarrow G \rightarrow 1$ eine exakte Sequenz von pro-endlichen Gruppen mit pro-p-Gruppe $H$ und $\left(x_{j}\right)_{j \in J}$ eine Familie von Elementen aus $H$; dann wird $H$ genau dann als Normalteiler in $E$ von den $x_{j}$ erzeugt, wenn $H^{\mathrm{ab}}$ als $\mathbb{Z}_{p} \llbracket G \rrbracket-$ Modul von den Restklassen $\bar{x}_{j}$ der $x_{j}$ in $H^{\mathrm{ab}}$ erzeugt wird.

Beweis. Sei $H^{\prime}$ der von den $x_{j}$ (topologisch) erzeugte Normalteiler. Nach dem Burnside-Satz für pro- $p$-Gruppen ist die Surjektivität der Inklusion $i: H^{\prime} \rightarrow H$ äquivalent zur Surjektivität der induzierten Abbildung $\bar{i}: H^{\prime a b} \rightarrow H^{\mathrm{ab}}$. Da $H^{\prime}$ die von allen Konjugierten der $x_{j}$ erzeugte abgeschlossene Untergruppe ist, ist $\operatorname{Im} \bar{i}$ gerade der von den $\bar{x}_{j}$ erzeugte $\mathbb{Z}_{p} \llbracket G \rrbracket$-Untermodul. q.e.d.

Um eine Aussage über die Relationen von $G_{k}$ zu erhalten, gehen wir nicht von einer Darstellung mittels einer freien Gruppe aus, sondern nutzen schon aus, was wir nach 3.1 über die Struktur von $G_{k}$ wissen. Genauer gesagt, konstruieren wir die freien Objekte der folgenden Kategorie $\mathcal{N}_{\mathscr{G}}^{p}$ : Objekte sind die pro-endlichen Gruppen $E$, die semidirektes Produkt von $\mathscr{G}$ mit einer pro-pGruppe $H$ sind, $E=H \cdot \mathscr{G}$, und Morphismen sind die stetigen Homomorphismen $f: H^{\prime} \cdot \mathscr{G} \rightarrow H \cdot \mathscr{G}$, die $\mathscr{G}$ elementweise festlassen und $H^{\prime}$ in $H$ abbilden.

Sei dazu $J$ eine Indexmenge und $F(J)$ die freie pro-endliche Gruppe mit freien Erzeugenden $z_{j}, j \in J$. Der Kern der kanonischen Projektion des freien pro-endlichen Produktes $F(J) * \mathscr{G}$ auf $\mathscr{G}$ ist gerade der von den $z_{j}$ erzeugte Normalteiler $Z=\left\langle z_{j} \mid j \in J\right\rangle$ (s. Neukirch [12], 1.2). Die gesuchte Gruppe erhalten wir nun, indem wir $Z$ zur pro- $p$-Gruppe machen, d.h., mit dem Normalteiler $N$ von $Z$, für den $Z / N=Z(p)$ die maximale pro- $p$-Faktorgruppe von $Z$ ist, setzen wir

$$
\begin{aligned}
F(J, \mathscr{G}) & =F(J) * \mathscr{G} / N, \\
P_{J, \mathscr{G}} & =Z / N=\left\langle z_{j} \mid j \in J\right\rangle(p)
\end{aligned}
$$

( $N$ ist auch normal in $F(J) * \mathscr{G})$. Offenbar ist $F(J, \mathscr{G})$ semidirektes Produkt von $\mathscr{G}$ mit $P_{J, \mathscr{G}}$ und damit aus $\mathcal{N}_{\mathscr{G}}^{p}$. Es ergibt sich nun leicht, daß $F(J, \mathscr{G})$ freies Objekt in $\mathcal{N}_{g}^{p}$ ist, und zwar frei auf der Familie $\left(y_{j}\right)_{j \in J}$ mit $y_{j}=z_{j} N \in P_{J, g}$.

Satz 3.4. a) $P_{J, \mathscr{G}}$ ist eine freie pro-p-Gruppe, und $P_{J, \mathscr{G}}^{\mathrm{ab}}$ ist ein freier $\mathbb{Z}_{p} \llbracket \mathscr{G} \rrbracket-M o d u l$ mit der Basis $\left\{\bar{y}_{j}=y_{j}\left[P_{J, \mathscr{B}}, P_{J, \mathscr{G}}\right] / j \in J\right\}$.

b) Ist $E=H \cdot G \in \mathcal{M}_{g}^{p}$ und $\left(x_{j}\right)_{j \in J}$ eine konvergente Familie von Elementen aus $H$ (d.h., in jedem offenen Normalteiler von $H$ liegen fast alle $\left.x_{j}\right)$, dann gibt es ein eindeutig bestimmtes $f: F(J, \mathscr{G}) \rightarrow E$ mit $f\left(y_{j}\right)=x_{j}$ für $j \in J$.

c) Ist unter den Voraussetzungen von b) $H$ eine freie pro-p-Gruppe und $H^{\mathrm{ab}}$ $\cong \mathbb{Z}_{p}[\mathscr{G}]^{J}$ mit Basis $\left\{\bar{x}_{j}=x_{j}[H, H] / j \in J\right\}$, so ist $f$ ein Isomorphismus.

Beweis. b) folgt daraus, daß es einen eindeutig bestimmten stetigen Homomorphismus $f_{0}: F(J) * \mathscr{G} \rightarrow E$ mit $f_{0}\left(z_{j}\right)=x_{j}$ für alle $j \in J$ und $f_{0}(\rho)=\rho$ für alle $\rho \in \mathscr{G}$ gibt, der sich über $N$ faktorisiert, da $H$ eine pro-p-Gruppe ist.

a) Wir setzen zur Abkürzung $F=F(J)$ und verwenden die vorher gewählten Bezeichnungen. Für einen endlichen $Z$-Modul $A$ ist der $F * \mathscr{G} / Z$-induzierte 
Modul $B=M_{F * \mathscr{G}}^{Z}(A)$ ein induzierter $\mathscr{G}$-Modul (isomorph zu $M_{\mathscr{G}}(A)$ ). Da die Restriktion

$$
H^{q}(Z, A) \cong H^{q}(F * \mathscr{G}, B) \stackrel{\text { res }}{\longrightarrow} H^{q}(\mathscr{G}, B)=0
$$

wegen $c d(F)=1$ ein Isomorphismus für $q \geqq 2$ ist (vgl. [12], Satz 4.2), folgt $c d(Z) \leqq 1$ und damit auch $c d_{p}\left(P_{J, G}\right)=c d_{p}(Z(p)) \leqq 1$. Wenden wir weiter b) auf die Gruppe $E^{\prime}=\left(\mathbb{Z}_{p} \llbracket \mathscr{G} \rrbracket^{J}\right) \cdot \mathscr{G} \in \mathscr{N}_{\mathscr{G}}^{p}$ an, so erhalten wir einen surjektiven $\mathbb{Z}_{p} \llbracket \mathscr{G} \rrbracket-$ Homomorphismus

$$
\bar{g}: P_{J, \mathscr{G}}^{\mathrm{ab}} \rightarrow \mathbb{Z}_{p} \llbracket \mathscr{G} \rrbracket^{J}=\prod_{j \in J} \mathbb{Z}_{p} \llbracket \mathscr{G} \rrbracket b_{j}
$$

mit $\bar{g}\left(\bar{y}_{j}\right)=b_{j}$. Da die $\bar{y}_{j}$ eine konvergente Familie bilden, gibt es einen stetigen $\mathbb{Z}_{p} \llbracket \mathscr{G} \rrbracket-$ Homomorphismus $\bar{h}$ in die umgekehrte Richtung mit $\bar{h}\left(b_{j}\right)=\bar{y}_{j}$ (vgl. [1], 1.2). Wegen $\bar{g} \bar{h}\left(b_{j}\right)=b_{j}$ ist $\bar{g} \bar{h}$ die Identität, ebenso muß nach der Eindeutigkeitsaussage in b) das Kompositum $\bar{h} \bar{g}$ die Identität sein.

c) Unter den genannten Voraussetzungen induziert $f$ einen Isomorphismus von $P_{J, \mathscr{a}}^{\mathrm{ab}}$ auf $H^{\mathrm{ab}}$, ist daher insbesondere surjektiv. Setzen wir $K=\operatorname{Ker} f$ und $H^{q}(X)=H^{q}(X, \mathbb{Z} / p \mathbb{Z})$ für eine pro-p-Gruppe $X$, so liegt $K$ in $P_{J, \mathscr{G}}$, und aus der exakten Sequenz

$$
0 \rightarrow H^{1}(H) \stackrel{f^{*}}{\sim} H^{1}\left(P_{J, \xi}\right) \rightarrow H^{1}(K)^{H} \rightarrow H^{2}(H)=0
$$

der niedrigen Terme der Spektralsequenz für $H=P_{J, \mathscr{G}} / K$ folgt $H^{\mathrm{t}}(K)^{H}=0$ und damit $K=1$, da $K$ und $H$ pro- $p$-Gruppen sind. q.e.d.

Bemerkung 3.5. In den obigen Ausführungen kann $\mathscr{G}$ durch eine beliebige proendliche Gruppe ersetzt werden. $P_{J, \mathscr{G}}$ ist gerade eine freie Operatoren-pro- $p$ Gruppe mit freiem Erzeugendensystem $\left\{y_{j}, j \in J\right\}$ und Operatorenbereich $\mathscr{G}$ in der Terminologie von Koch [10].

Wir können nun den angekündigten Satz über die Relationenanzahl von $G_{k}$ aussprechen. Für eine endliche Indexmenge $J=\{1, \ldots, m\}$ schreiben wir dabei $F(m, \mathscr{G})$ statt $F(J, \mathscr{G})$, weiter fassen wir vermöge eines fest gewählten Schnittes, der nach 3.1 existiert, $\mathscr{G}$ als Untergruppe von $G_{k}$ auf.

Satz 3.6. Ist $k$ eine endliche Erweiterung von $\mathbb{Q}_{p}$, so gilt mit $n=\left[k: \mathbb{Q}_{p}\right]$ :

a) Enthält $T$ keine primitive p-te Einheitswurzel, so ist $G_{k}$ isomorph zu $F(n, \mathscr{G})$.

b) Enthält T eine primitive p-te Einheitswurzel, so gibt es eine Surjektion

$$
F(n+1, \mathscr{G}) \rightarrow G_{k},
$$

deren Kern als Normalteiler von einem Element $r$ erzeugt wird. Zusatz: Sind $x_{0}, \ldots, x_{n} \in V_{k}$ Liftungen der $\mathbb{Z}_{p} \llbracket \mathscr{G} \rrbracket$-Erzeugenden $\bar{x}_{0}, \ldots, \bar{x}_{n}$ von $V_{k}^{\mathrm{ab}}$ aus Satz 3.2, ist $F(n+1, \mathscr{G})$ frei auf $y_{0}, \ldots, y_{n}$ und $f: F(n+1, \mathscr{G}) \rightarrow G_{k}$ der Homomorphismus mit $f\left(y_{j}\right)=x_{j}, f(\sigma)=\sigma$ und $f(\tau)=\tau$, so kann mit den Bezeichnungen aus 3.2

$$
r \equiv y_{0}^{-\sigma} y_{0}^{g \lambda} y_{1}^{p^{s}} \bmod \left[P_{n+1, \mathscr{G}}, P_{n+1, \mathscr{G}}\right]
$$

gewählt werden (hier ist $P_{n+1, \mathscr{G}}$ der Kern der kanonischen Projektion $F(n$ $+1, \mathscr{G}) \rightarrow \mathscr{G})$. 
Beweis. a) folgt aus $3.4 \mathrm{c}$ ) und $3.2 \mathrm{a}$ ), da $V_{k}$ eine freie pro-p-Gruppe ist.

b) Wählen wir $f$ wie im Zusatz, setzen wir $N=\operatorname{Ker} f$ und zur Abkürzung $P$ $=P_{n+1, \mathscr{G}}$, so erhalten wir ein kommutatives Diagramm

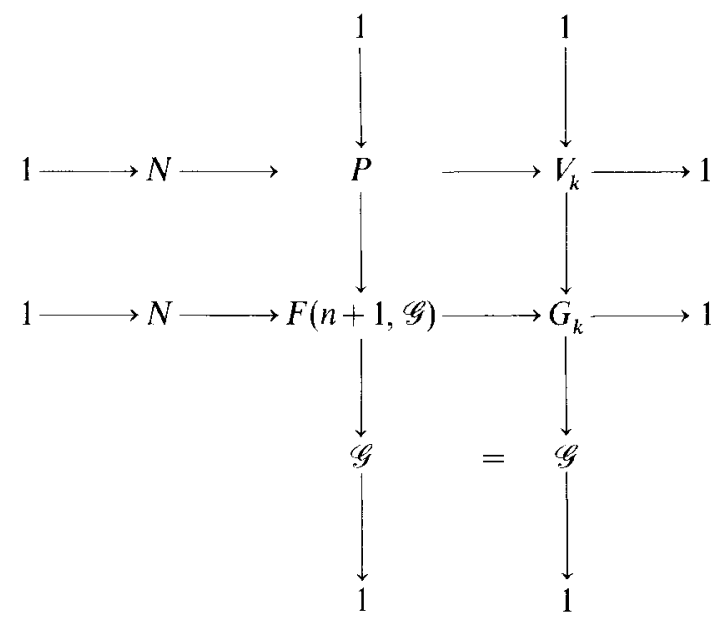

mit exakten Zeilen und Spalten. Aus der Spektralsequenz für die obere Zeile erhalten wir wegen $c d_{p}\left(V_{k}\right)=1$ die exakte Sequenz

$$
0 \rightarrow H^{1}\left(V_{k}, \mathbb{Q}_{p} / \mathbb{Z}_{p}\right) \rightarrow H^{1}\left(P, \mathbb{Q}_{p} / \mathbb{Z}_{p}\right) \rightarrow H^{1}\left(N, \mathbb{Q}_{p} / \mathbb{Z}_{p}\right)^{P} \rightarrow 0
$$

und dual dazu die exakte Sequenz von $\mathbb{Z}_{p} \llbracket \mathscr{G} \rrbracket-$ Moduln

$$
0 \rightarrow N /[N, P] \rightarrow P^{\mathrm{ab}} \rightarrow V_{k}^{\mathrm{ab}} \rightarrow 0 .
$$

Wegen $P^{\mathrm{ab}}=\bigoplus_{j=0}^{n} \mathbb{Z}_{p} \llbracket \mathscr{G} \rrbracket \bar{y}_{j}$ und $f\left(\bar{y}_{j}\right)=\bar{x}_{j}$ (wobei $\bar{y}_{j}=y_{j}[P, P]$ gesetzt ist) ergibt ein Vergleich mit Satz 3.2

$$
N /[N, P] \cong \mathbb{Z}_{p} \llbracket \mathscr{G} \rrbracket .
$$

Ist $r \in N$ derart gewählt, daß $r[N, P]$ den $\mathbb{Z}_{p} \llbracket \mathscr{G} \rrbracket$-Modul $N /[N, P]$ erzeugt, so erzeugt $r[N, N]$ auch $N^{\text {ab }}$ als $\mathbb{Z}_{p} \llbracket G_{k} \rrbracket$-Modul. Dies folgt aus dem NakayamaLemma: $\mathbb{Z}_{p} \llbracket P \rrbracket$ ist ein lokaler Ring, und der Kern $I_{P}$ der kanonischen Projektion von $\mathbb{Z}_{p} \llbracket P \rrbracket$ auf $\mathbb{Z}_{p}$ ist im maximalen Ideal von $\mathbb{Z}_{p} \llbracket P \rrbracket$ enthalten (da $P$ eine pro-p-Gruppe ist, vgl. [1]); der $\mathbb{Z}_{p} \llbracket G_{k} \rrbracket$-Homomorphismus

$$
\varphi: \mathbb{Z}_{p}\left[G_{k} \rrbracket \rightarrow N^{\mathrm{ab}} \quad \varphi(1)=r[N, N]\right.
$$

ist daher genau dann surjektiv, wenn die induzierte Abbildung

$$
\bar{\varphi}: \mathbb{Z}_{p} \llbracket G_{k} \rrbracket / I_{p} \mathbb{Z}_{p} \llbracket G_{k} \rrbracket \cong \mathbb{Z}_{p} \llbracket \mathscr{G} \rrbracket \rightarrow N^{\mathrm{ab}} / I_{P} N^{\mathrm{ab}}=N /[N, P]
$$

surjektiv ist. Schließlich erzeugt ein derartiges $r$ nach Lemma 3.3 auch $N$ als Normalteiler in $F(n+1, \mathscr{G})$. Die übrigen Aussagen sind klar. 


\section{B) Potenzreihenkörper}

Die entsprechenden Ergebnisse für Potenzreihenkörper lassen sich folgendermaßen neu formulieren.

Satz 3.7 (Koch [11]). Sei $k=\mathbb{F}_{q}((X))$ der Körper der formalen Laurentreihen mit Koeffizienten im Körper $\mathbb{F}_{q}$ mit $q=p^{f_{0}}$ Elementen.

a) Für eine endliche, galoissche, zahm-verzweigte Erweiterung $K / k$ mit Galoisgruppe $G$ gilt die $\mathbb{Z}_{p}[G]$-Isomorphie

$$
U_{K}^{1} \cong \mathbb{Z}_{p}[G]^{\mathbb{N}} .
$$

Dies ist ein topologischer Isomorphismus, wenn man rechts die Produkttopologie nimmt.

b) Es gilt die $\mathbb{Z}_{p} \llbracket \mathscr{G} \rrbracket$-Isomorphie $V_{k}^{\mathrm{ab}} \cong \mathbb{Z}_{p} \llbracket \mathscr{G} \rrbracket^{\mathrm{N}}$, und $G_{k}$ ist isomorph zu $F(\mathbb{N}, \mathscr{G})$.

Beweis. a) Ist $e_{K}$ der Verzweigungsindex von $K / k$ und $U_{K}^{i}$ die Gruppe der Einseinheiten $i$-ter Stufe in $K$, so gilt nach einem Lemma von Iwasawa ([7], Lemma 1 und [11], Beweis von Satz 1) die Isomorphie

$$
U_{K}^{1} / U_{K}^{p e_{K} j}\left(U_{K}^{1}\right)^{p} \cong \mathbb{F}_{p}[G]^{(p-1) f_{0} j} .
$$

Durch Übergang zum projektiven Limes über $j$ erhält man

$$
U_{K}^{1} /\left(U_{K}^{1}\right)^{p} \cong \mathbb{F}_{p}[G]^{\mathbb{N}},
$$

woraus a) wegen der Torsionsfreiheit von $U_{K}^{1}$ folgt.

b) Für zahm-verzweigtes $K^{\prime} / K$ ist $N_{K^{\prime} / K}\left(U_{K^{\prime}}^{p^{e^{\prime}}{ }^{\prime} j}\right)=U_{K}^{p_{K} j}$, daher gilt

$$
\begin{aligned}
& V_{k} / V_{k}^{p}\left[V_{k}, V_{k}\right] \cong \lim _{\substack{K \subset T \\
K / k \text { endl.gal. }}} U_{K}^{1} /\left(U_{K}^{1}\right)^{p} \cong \lim _{K \in T} \lim _{j} U_{K}^{1} / U_{K}^{p e_{K} j}\left(U_{K}^{1}\right)^{p} \\
& \cong \lim _{j}{\underset{\lim }{K \subset T}}_{\mathbb{F}_{p}}[G(K / k)]^{(p-1) f_{0} j} \cong \varliminf_{j}^{\lim } \mathbb{F}_{p} \llbracket \mathscr{G} \rrbracket^{(p-1) f_{0} j} \cong \mathbb{F}_{p} \llbracket \mathscr{G} \rrbracket^{\mathbb{N}} .
\end{aligned}
$$

Wegen der Torsionsfreiheit von $V_{k}^{\text {ab }}$ ist also $V_{k}^{\text {ab }} \cong \mathbb{Z}_{p} \llbracket \mathscr{G} \rrbracket^{\mathbb{N}}$. Die Isomorphie $G_{k}$ $\cong F(\mathbb{N}, \mathscr{G})$ folgt nun mit Satz $3.4 \mathrm{c})$, denn $V_{k}$ ist eine freie pro-p-Gruppe, und mit Hilfe eines stetigen Schnittes der Abbildung $V_{k} \rightarrow V_{k}^{\text {ab }}$ erhält man eine konvergente Familie $\left(x_{j}\right)_{j \in \mathbb{N}}$, deren Bild eine $\mathbb{Z}_{p} \llbracket \mathscr{G} \rrbracket$-Basis von $V_{k}^{\text {ab }}$ ist.

\section{Literatur}

1. Brumer, A.: Pseudocompact algebras, profinite groups, and class formations. J. Algebra 4, 442$470(1966)$

2. Cartan, H., Eilenberg, S.: Homological algebra. Princeton 1956

3. Gilbarg, D.: The structure of the groups of p-adic 1-units. Duke Math. J. 9, 262-271 (1942)

4. Gruenberg, K.W.: Cohomological topics in group theory. Lect. Notes in Math. vol. 143. BerlinHeidelberg-New York: Springer 1970 
5. Gruenberg, K.W.: Relation modules of finite groups. Conference board of the math. sciences, vol. 25. AMS 1976

6. Hasse, H.: Zahlentheorie. Berlin: Akademie Verlag 1963

7. Iwasawa, K.: On galois groups of local fields. Trans. Amer. Math. Soc. 80, 448-469 (1955)

8. Jakovlev, A.V.: The galois group of the algebraic closure of a local field. Math. USSR-Izv. 2, 1231-1269 (1968); Remarks on my paper "The galois group of the algebraic closure of a local field". Math. USSR-Izv. 12, 205-206 (1978)

9. Jannsen, U., Wingberg, K.: Die Struktur der absoluten Galoisgruppe p-adischer Zahlkörper. Invent. math. 70, 71-98 (1982)

10. Koch, H.: Über Galoissche Gruppen von p-adischen Zahlkörpern. Math. Nachr. 29, 77-111 (1965)

11. Koch, H.: Über die Galoissche Gruppe der algebraischen Abschließung eines Potenzreihenkörpers mit endlichem Konstantenkörper. Math. Nachr. 35, 323-327 (1967)

12. Neukirch, J.: Freie Produkte pro-endlicher Gruppen und ihre Kohomologie. Arch. d. Math. (Basel) 12, 337-357 (1971)

13. Pieper, H.: Die Einseinheitengruppe eines zahm-verzweigten galoisschen lokalen Körpers als Galois-Modul. Math. Nachr. 54, 173-210 (1972)

14. Serre, J-P.: Corps locaux. Paris: Hermann 1962

15. Serre, J-P.: Cohomologie galoisienne. Lect. Notes in Math., vol. 5. Berlin-Heidelberg-New York: Springer 1973

16. Swan, R.G.: Induced representations and projective modules. Ann. of Math. 71, 552-578 (1960) 